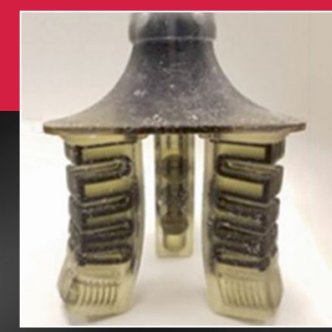

\title{
Three-dimensional printing of tactile sensors for soft robotics
}

\section{Xinran Zhou and Pooi See Lee*}

Three-dimensional (3D) printing has become an important fabrication method for soft robotics, due to its ability to make complex 3D structures from computer designs in simple steps and multimaterial co-deposition ability. In this article, the application of 3D printing techniques in the fabrication of four types of tactile sensors commonly used in soft robotics, including the piezoresistive tactile sensor, capacitive tactile sensor, piezoelectric tactile sensor, and triboelectric tactile sensor, will be discussed. The 3D printing mechanism, material, and structure for each type of sensor will be introduced, and the perspectives on the future potential of 3D printable tactile sensors will be discussed.

\section{Introduction}

Different from traditional robots made of rigid materials, soft robots rely on highly compliant materials to be flexible and adaptable to move and work in complex environments and accomplish unique tasks. Sensors are one of the essential components in soft robotics, which include tactile sensors, motion sensors, biological sensors, and chemical sensors. Tactile sensors are one of the most important sensors for soft robotics since they can perceive the physical interaction with the environment, and the feedback information can be used to guide the robot to achieve more accurate motions such as grasping or movement. Tactile sensors can be manipulated by mechanical factors such as force, pressure, strain, stiffness, roughness, and friction, as well as non-mechanical factors such as temperature from the environment.

Traditional tactile sensing includes four types: piezoresistive sensing, capacitive sensing, piezoelectric sensing, and optical sensing. In piezoresistive sensing, the resistivity of the sensor changes with the mechanical deformation. Similarly, in capacitive sensing, the capacitance of the sensing material changes with deformation. Piezoresistive sensing is robust against electrical noise, but the frequency response is relatively low. ${ }^{1}$ Capacitive sensing has good frequency response, high spatial resolution, and large dynamic ranges, but it can be affected by multiple types of noises. ${ }^{2}$ Different from piezoresistive and capacitive sensing, piezoelectric sensing can be self-powered by the direct piezoelectric effect originated from the internal polarization under external stress. It can also function with an external power supply through converse piezoelectric effect, by actuating the sensor to vibrate under the resonance frequency, and the resonance frequency shifts under stress. Piezoelectric sensing exhibits a very high frequency response, but low spatial resolution. ${ }^{2}$ In recent years, triboelectric effect was also considered for tactile sensing application. Similar to piezoelectric effect, triboelectricity can be understood by the displacement part of Maxwell equations, and it generates electric signals by contact electrification and electrostatic induction without the need of a power supply. ${ }^{3}$ Optical sensors analyze the changes in modulation of wavelength, phase, polarization, or intensity of the light under deformation of the waveguide. ${ }^{1}$ Since the mechanism and material system of optical sensing is largely different from the other four sensing principles, in this article, we will focus on piezoresistive, capacitive, piezoelectric, and triboelectric sensing, which are electronic-based sensing.

Different from traditional robotics with rigid assembly of devices, soft robotics prefer to cast all the parts together without assembly, or assemble by adhesive. As a rapid prototyping

Xinran Zhou, School of Materials Science and Engineering, Nanyang Technological University, and Singapore-HUJ Alliance for Research and Enterprise, Nanomaterials for Energy and Water Nexus, Campus for Research Excellence and Technological Enterprise, Singapore; xrzhou@ntu.edu.sg

Pooi See Lee, School of Materials Science and Engineering, Nanyang Technological University, and Singapore-HUJ Alliance for Research and Enterprise, Nanomaterials for Energy and Water Nexus, Campus for Research Excellence and Technological Enterprise, Singapore; pslee@ntu.edu.sg 
method, 3D printing becomes increasingly popular in the fabrication of soft robotics. Compared to the traditional casting method, 3D printing directly forms the required structure with less time and material consumption, and can fabricate more complex structures. In this article, we will introduce the principle, progress, and potential of 3D printed piezoresistive, capacitive, piezoelectric, and triboelectric tactile sensors for soft robotics application (Figure 1).
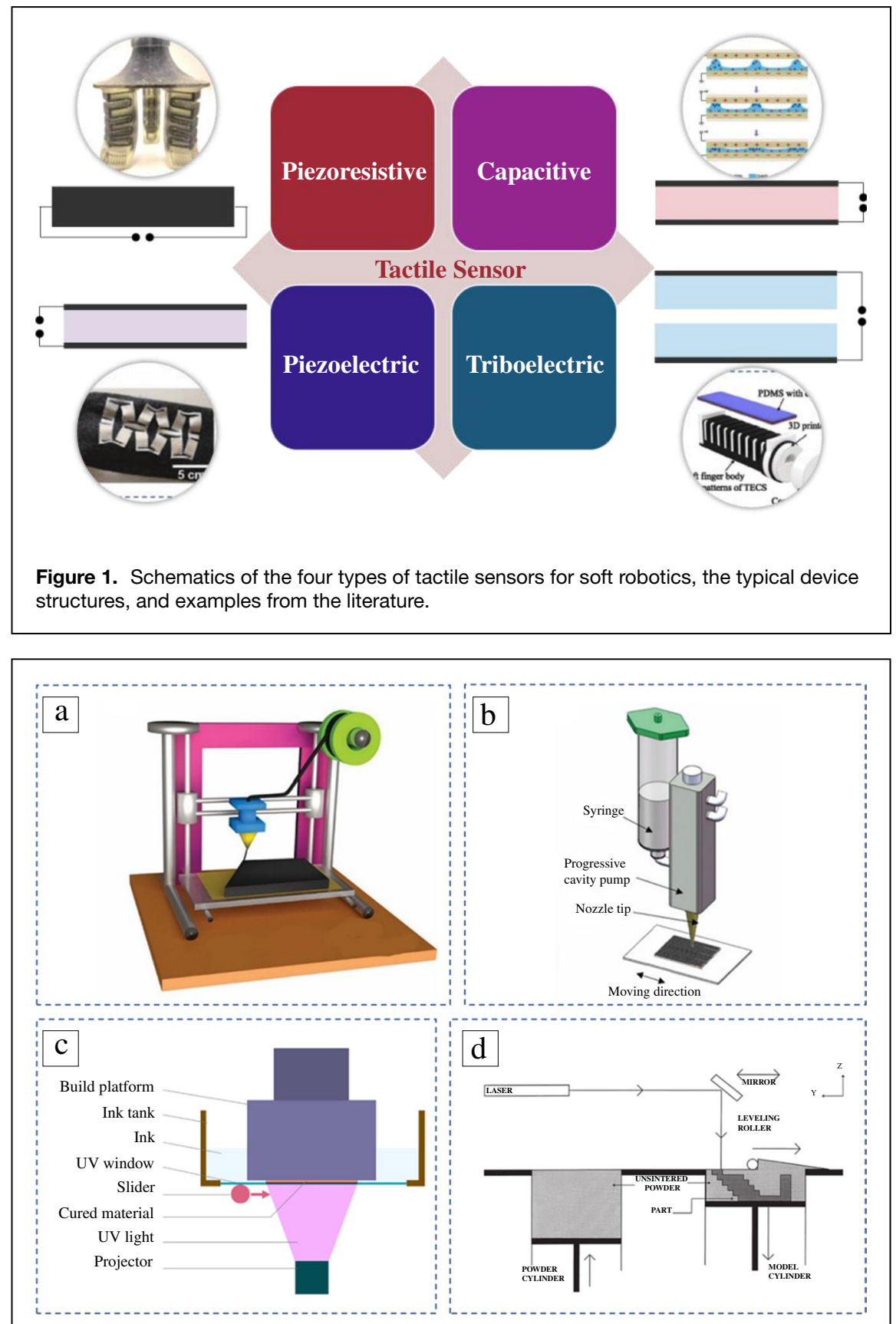

Figure 2. Schematics of general printing methods: (a) Fused deposition modeling. Reprinted with permission from Reference 8. () 2017 Wiley. (b) Direct ink writing. Reprinted with permission from Reference 9. () 2019 Elsevier. (c) Stereolithography. ${ }^{10}$ (d) Selective laser sintering. Reprinted with permission from Reference 11. (c) 1998 Elsevier.

\section{Three-dimensional printing materials and mechanisms}

In tactile sensor fabrication, 3D printing can be applied in depositing conductive materials such as the electrodes and piezoresistive materials, as well as functional materials such as dielectric polymers, piezoelectric materials, and triboelectric materials. At present, various 3D printing techniques are used for tactile sensor fabrication, among which, the two most frequently used techniques are fused deposition modeling (FDM, also known as fused filament fabrication $[F F F]$ ), and direct ink writing (DIW). In FDM, thermoplastics or composites with thermoplastic matrices are made into filaments. During printing, the filament is heated to around $200^{\circ} \mathrm{C}$ above the glass transition temperature and softens at the printing head to be extruded to a relatively cold platform. The extruded filament immediately solidifies to retain the shape (Figure 2a). FDM is the simplest, cheapest, most accessible, and environmentally friendly $3 \mathrm{D}$ printing method. However, it has limited printable materials, and the resolution is usually relatively low compared to other printing methods. In contrast, DIW is based on an extrusion and curing process, which is suitable for a wide range of materials, from solution with extremely low viscosity to plasticine-like material with very high viscosity (Figure 2b). However, for best printing results, the material should have shear-thinning behavior so that after extrusion, it can retain its shape without collapsing. The commonly used curing methods for DIW are thermal curing, solvent evaporation, and UV curing. Both DIW and FDM can achieve multimaterial printing by using multiple printing heads. Other 3D printing methods that are used to fabricate tactile sensors include digital light processing (DLP), material jetting (MJ) 3D printing, and selective laser sintering (SLS). Both DLP and $\mathrm{MJ}$ require the materials to be UV curable. In DLP, UV-curable ink is loaded in a tank with transparent 
Teflon film as the bottom UV window (Figure 2c). The building platform is placed upside down slightly above the bottom of the ink tank, and UV light will be projected through the UV window to cure thin layers of ink onto the building platform. The resolution for DLP printing (tens of micrometers) is usually higher than FDM and DIW, and since the printing is upside down, no supporting material is needed to form complex structures. However, normal DLP printers are not suitable for multimaterial printing. In some recent research on $3 \mathrm{D}$ printed tactile sensors, the MJ method is used. In this method, materials are deposited by MJ and UV curing layer by layer. This is not a traditional 3D printing method and limited commercial printers are available. Noticeably, almost all of the current research on MJ 3D printing of tactile sensors use printers from the same company except one, who use a piezoelectric-pneumatic printhead; ${ }^{4}$ while these commercial printers only support limited commercial materials from the same company (including various rigid, elastic, and conductive polymers), the exact composition of the material is unknown..$^{5-7}$ The commercial MJ printers can print multiple materials at the same time, and by using this printer, the sensors can be easily commercialized since the material and fabrication method is standardized and can achieve high resolution. A few reports also use SLS to fabricate tactile sensors, SLS uses thermal energy from the laser to selectively melt and bind metal, ceramic, or thermoplastic powders to build a 3D structure (Figure 2d). SLS can achieve isotropic mechanical properties in the printed object and no supporter materials are needed. However, it generally requires a relatively large amount of powder material, which serves as the powder bed to support the printed structure. It is also not able to build multimaterials at one time. Overall, printing methods can be selected according to the type of materials, resolution requirement, and multimaterial fabrication requirement.
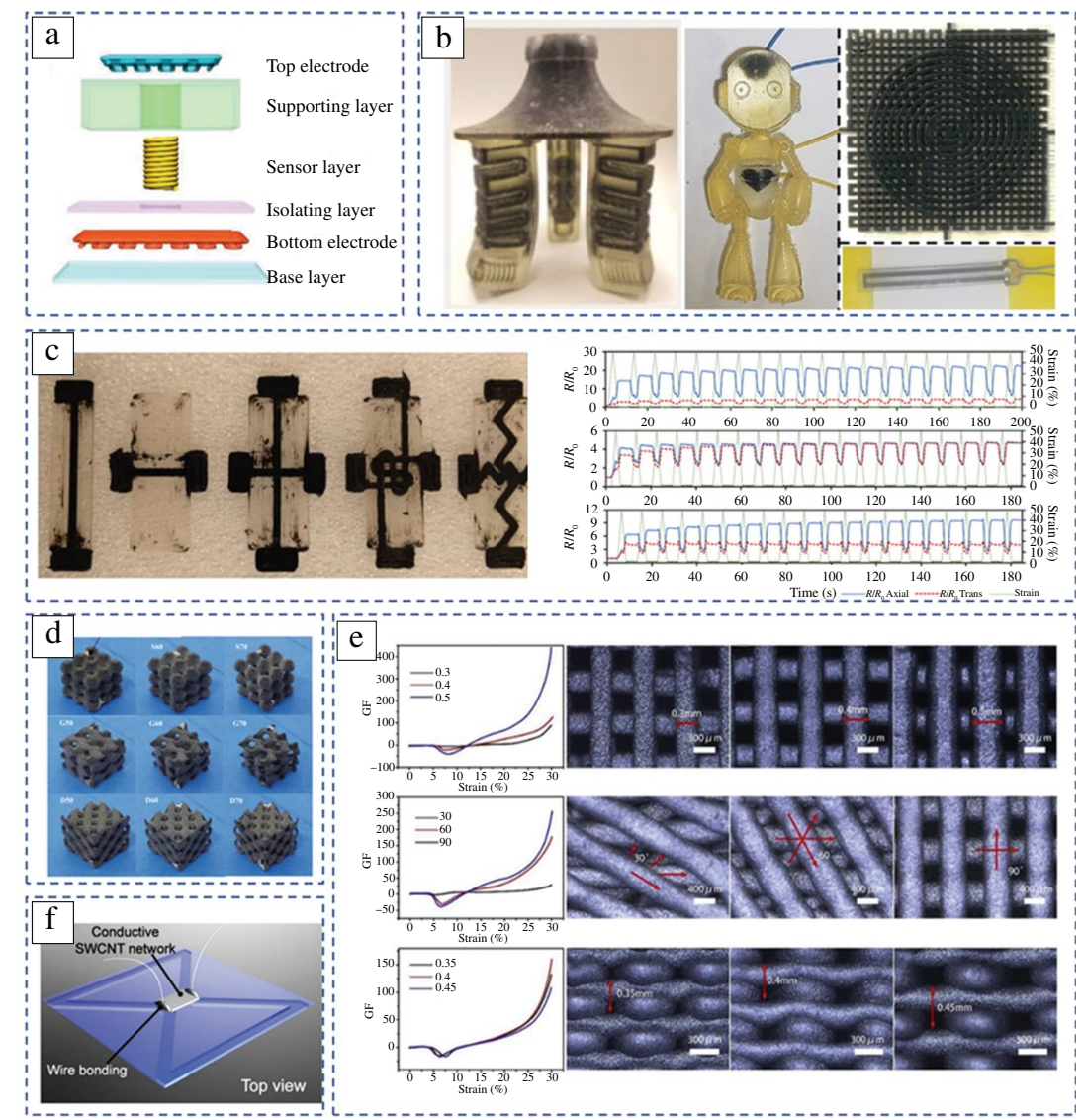

Figure 3. Three-dimensional printed piezoresistive nanogenerators. (a) An all-3D printed stretchable tactile sensors. Reprinted with permission from Reference 12. (c) 2017 Wiley. (b) Three-dimensional printed, piezoresistive sensors embedded in soft robotics. Reprinted with permission from Reference 5. (C) 2019, Shih, Christianson, Gillespie, Lee, Mayeda, Huo, and Tolley. (c) Biaxial sensors with different conductive pattern designs. ${ }^{13}$ (d) Selective laser sintering fabricated piezoresistive sensors with minimal surface-based porous structure. ${ }^{14}$ (e) Gauge factor (GF) affected by filament diameters, interaxial angles, and interlayer spaces. Reprinted with permission from Reference 15. (C) 2018 Elsevier. (f) A 3D printed auxetic structure-assisted piezoresistive sensor. Reprinted with permission from Reference 16. (C) 2018 Wiley.

\section{Three-dimensional printed piezoresistive sensors}

For piezoresistive sensing, sensing materials consist of conducting or semiconducting materials, of which the resistivity change with applied mechanical strain. In recent years, carbon-based materials have been widely used as piezoresistive materials, as they are stable, have physical properties that offer higher sensitivity and selectivity, and can be composited with elastic polymers such as polystyrene (PS), polyurethane (PU), and poly(dimethylsiloxane) (PDMS) for adjustable conductivity. ${ }^{1}$ All of these substrate material systems can be 3D printed with different methods. For example, thermoplastic polyurethane (TPU), poly(lactic acid) (PLA), and poly(acrylonitrile butadiene styrene) (ABS) systems can be printed by FDM; thermosetting elastomers systems such as silicone, polyurethane, and PDMS can be printed with DIW; and polyurethane acrylate (PUA) systems can be printed with DLP.

By 3D printing, multimaterial deposition can be achieved within a single printing step. For example, Guo et al. developed a series of room-temperature curable inks and used the DIW technique to print the whole piezoresistive tactile sensor, including the silicone 
as a base layer and isolation layer; different ratios of $\mathrm{Ag} /$ silicone layers as electrodes and sensor layers; and watersoluble sacrificed supporting layers (Figure 3a). ${ }^{12}$ The sensor is fabricated in one printing step to be multimaterial, multiscale, and multifunctional. This flexible and stretchable sensor is capable of detecting human movements, including radial pulse, and finger pressing and bending. In addition to 3D printing the whole sensor, Shih et al. used a commercial MJ 3D printer and commercial inks to co-fabricate the sensor with a soft robot, which could help improve both the exteroceptive and interoceptive capabilities of the sensor comparing with non-embedded sensors (Figure $3 b$ ). ${ }^{5}$

Comparing this to traditional fabrication methods, the largest advantage for $3 \mathrm{D}$ printing is the capability of making complex macrostructures, which could affect the sensing performance by influencing the strain on the sensor during deformation. Christ et al. developed a bidirectional stretchable piezoresistive sensor by multimaterial FDM ${ }^{17}$ (Figure $3 \mathrm{c}$ ). Different conductive patterns were designed to provide differentiable sensitivity. For example, the sensor with a switchback biaxial pattern demonstrates nearly identical piezoresistivity $\left(R / R_{0}\right)$ in the axial and transverse directions, while simple biaxial and sawtooth biaxial pattern devices demonstrate much higher $R / R_{0}$ in axial direction than transverse direction. In addition to the two-dimensional conductive pathway, 3D structures of the printed piezoresistive material also affect the sensing behavior. Gan et al. reported a single-wall carbon nanotube (SWCNT)/TPU-based 3D piezoresistive sensor with both conductive segregation network microstructure and minimal surface porous macrostructure by SLS printing of SWCNT-coated TPU powders ${ }^{14}$ (Figure 3d). They have proven that among Schwarz, Gyroid, and Diamond structures, Schwarz structure leads to a much higher gauge factor than the other structures due to the largest internal deformation with the same overall compression displacement. Not only can the designed structure of piezoresistive materials affect the sensitivity, the same overall structure with different printing paths can also result in different sensitivities. Huang et al. reported a graphene/PDMS piezoresistive sensor by DIW $^{15}$ (Figure 3e). It has been proven that diameter of printed filaments, interaxial angle, and interlayer space all affect the gauge factor of the overall device. The gauge factor increases with the increasing diameter of printed filaments due to the relatively less conductive pathway in the larger diameter filament with comparatively uncompacted structure and lower contact area between the graphene sheets. The gauge factor increases with the larger interaxial angle of the printed filaments between layers, and smaller interlayer distances for the same reason. By 3D printing, mechanical metamaterials such as auxetic structures with a negative Poisson's ratio can be fabricated, which also helps to improve the performance of piezoresistive sensors. Jiang et al. reported an auxetic structure-assisted piezoresistive sensor by attaching a DLP fabricated auxetic structure on a SWCNT-based piezoresistive material ${ }^{16}$ (Figure $3 f$ ). The auxetic structure can expand in both axial and transverse directions when it is stretched in the axial direction, which can reduce the Poisson compression that squeezes the active materials together under deformation, and therefore, the active materials are stretched in both directions and the change in resistivity is maximized. It was shown that with the auxetic structure, the sensitivity is enhanced and the noise is reduced.

Three-dimensional printing techniques can also be modified or integrated with other techniques to produce more complex structures for piezoresistive tactile sensors; some examples are the core-shell 3D printing with a specially designed syringe to produce both a protective sheath layer and inner sensing material, ${ }^{17}$ plasma treatment after printing to create microstructure for both strain and gas sensing application, ${ }^{18}$ casting with $3 \mathrm{D}$ printed mold to create porous structure, ${ }^{19}$ and dip coating with ionic conducting hydrogel after 3D printing to achieve high transparency and mechanical properties such as large elongation at break, and good shape recovery. ${ }^{20}$

\section{Three-dimensional printed capacitive sensors}

Capacitive sensors are composed of dielectric materials and conductive electrode materials. The basic structure of capacitive sensors is parallel plate capacitors. Recently, elastomers such as PDMS, silicones, and TPU are used as the dielectric material of capacitive tactile sensors for elasticity and good chemical stability for soft robotic applications. The popular electrode materials for capacitive tactile sensors are carbon/ polymer composites and metal/polymer composites, while ionic conductive electrodes and metals can also be used as electrodes. As discussed in the 3D printed piezoresistive sensors section, most of these materials are suitable for 3D printing.

For multimaterial 3D printing of integrated soft robot, Hohimer et al. reported an FDM-printed pneumatic actuator integrated with built-in capacitive or piezoresistive sensors ${ }^{21}$ (Figure 4a). The dielectric material for capacitive sensors is TPU, and the electrodes are made of multiwall carbon nanotubes (MWCNTs)/TPU. Not only can the capacitive sensorintegrated pneumatic actuator attain self-feedback of its actuating curvature, it can also sense if it is in contact with other objects, which helps in the accurate grasping or motion of the potential pneumatic soft robotics. Ntagios et al. have printed a robot hand with embedded capacitive sensors with multiple materials by both FDM and DIW. ${ }^{22}$ TPU was printed with FDM as the dielectric material, while graphite ink was printed with DIW as the electrode. Although the robot hand in this research is rigid, the sensing components are flexible and suitable for soft robotic applications. The tightly integrated sensors within the $3 \mathrm{D}$ printed robotics could pave the way for producing robust, multifunctional, and cost-effective soft robotics within simple steps.

The macrostructures created by $3 \mathrm{D}$ printing can also affect the sensing behavior of capacitive sensors. Tuna et al. reported a capacitive pressure sensor with corrugated surface printed with a commercial thermoset material by a commercial MJ 3D printer as the dielectric material, and sputtered 


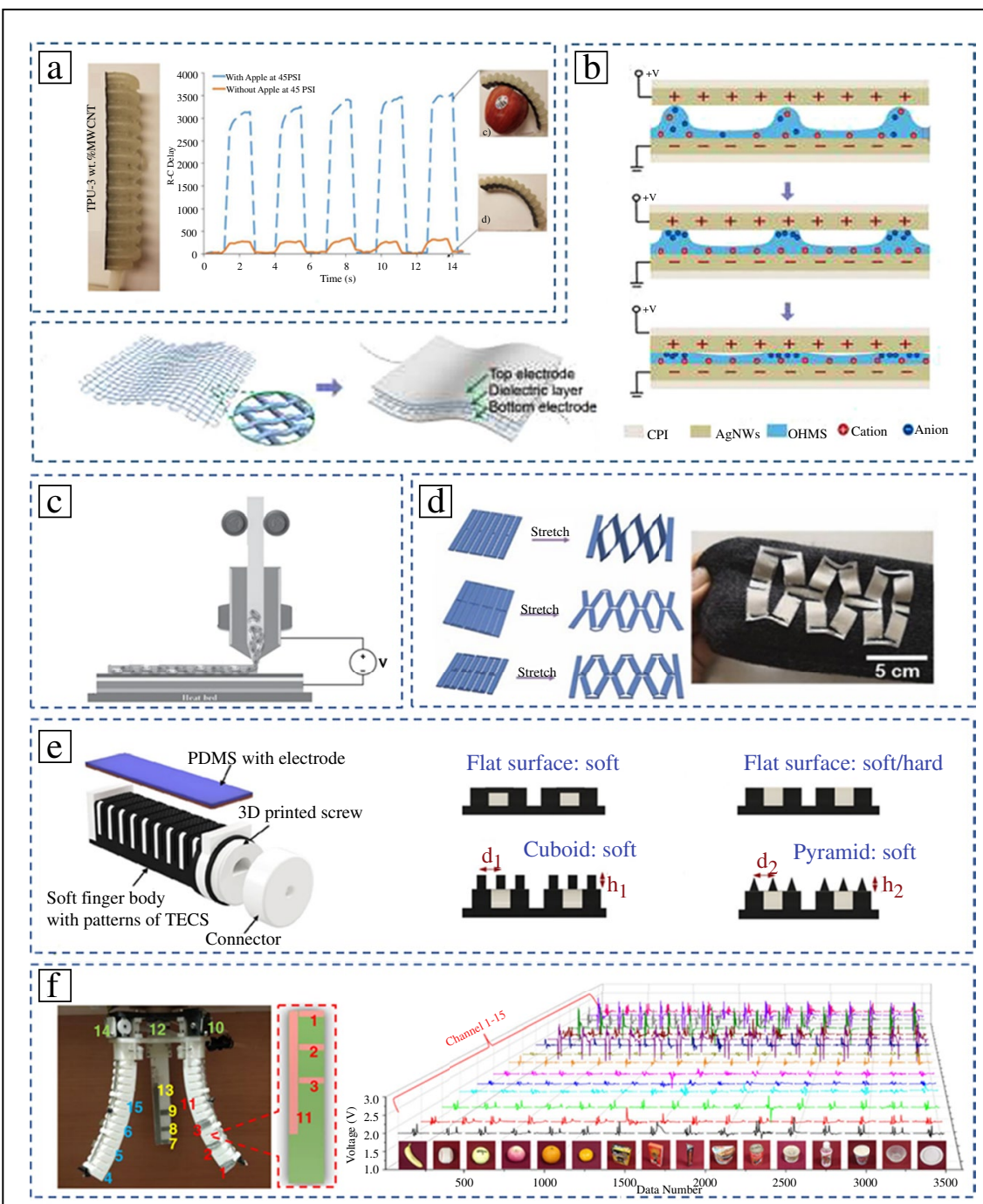

Figure 4. (a) A soft finger integrated with a capacitive sensor. Reprinted with permission from Reference 21. () 2020 Elsevier. (b) Tunable ionic capacitive sensor based on 3D printed mesh. Reprinted with permission from Reference 23. () 2020 Elsevier. (c) Illustration of in situ poling fused deposition modeling of piezoelectric material. Reproduced with permission from Reference 28. (c) 2017 Wiley. (d) Three-dimensional printed modified-kirigami piezoelectric sensor (TECS). ${ }^{29}$ (e) Three-dimensional printed soft finger with triboelectric curvature sensor. Reprinted with permission from Reference 6. () 2020 Elsevier. (f) Three-dimensional printed soft robot with triboelectric sensors for recognition of objects. ${ }^{32}$

$(<0.4 \mathrm{kPa})$ and broad pressure sensing range $(<400 \mathrm{kPa})$. The capacitive sensors with flat non$3 \mathrm{D}$ printed dielectric layer and patterned 3D printed electrode both showed enhancement in sensitivity. Lei et al. demonstrated a skinlike bifunctional stress and thermal sensor with hydrogel-based ionic conductors by DIW as electrodes and PE film as a dielectric layer. ${ }^{24}$ Comparing the low sensitivity with a flat electrode $\left(0.09 \mathrm{kPa}^{-1}\right)$, the pressure sensitivity of the gridstructured 3D printed hydrogel was up to $0.45 \mathrm{kPa}^{-1}$. The reason for the improved sensitivity was not provided; we postulated that the nodes in the grid-structured 3D printed electrode may concentrate the external pressure and generate a large local deformation on the dielectric layer. In addition to the regular 3D printing processes, coaxial printing ${ }^{25}$ and fiber-encapsulation additive manufacturing (FEAM) are also applied in fabricating capacitive tactile sensors. ${ }^{26}$

\section{Three-dimensional printed piezoelectric sensors}

With similar basic structure as the capacitive sensors, self-powered piezoelectric tactile sensors are composed of piezoelectric materials and two conductive electrodes. They are suitable for the small soft robots that do not have external power supply. Piezoelectric polymers and composites are deemed to be more suitable for soft robotic applications than the traditional rigid piezoelectric ceram-

$\mathrm{Cr}$ and $\mathrm{Au}$ as electrode. ${ }^{7}$ The pyramid-shaped corrugated surface of the capacitive sensor could enlarge the surface area by $19.6 \%$ so that the sensitivity is increased compared with the plain surface. Besides the designed structure, the structure formed automatically by the printing path is also valuable. Zou et al. fabricated an ionic capacitive sensor with DIW printed poly(vinylidene fluoride-co-hexafluoropropylene) (PVDF-HFP)-based ionic gels as dielectric material and $\mathrm{Ag} /$ polyimide films as electrodes ${ }^{23}$ (Figure 4b). The knots at the crossing of the axial and transverse printing paths form microdomes that deform and generate different internal ions' distribution under pressure, which exhibits a high sensitivity of $72.86 \mathrm{kPa}^{-1}$ in the ultralow pressure range ics-based sensors. Poly(vinylidene fluoride) (PVDF) family polymers are the piezoelectric polymers with the highest piezoelectricity. They can be 3D printed using solvent-evaporation DIW or the FDM method. Recently, elastomer composites with piezoelectric ceramic nanoparticle fillers are popular in fabricating sensors with elasticity. For example, Zhou et al. reported a stretchable piezoelectric sensor with a photocurable barium titanate (BTO) nanoparticle/PUA composite by DLP. ${ }^{10}$ However, since the $3 \mathrm{D}$ printable piezoelectric materials are not single crystals, they require an additional poling step to align the internal dipoles. This poling step can be combined with the FDM or DIW process by applying voltage between the extrusion needle and the platform (Figure 4c). Lee et al. reported the in situ poling 
method in FDM to deposit PVDF piezoelectric materials, ${ }^{27}$ and Kim et al. used the same method to fabricate a BTO/PVDF piezoelectric composite for pressure sensor application. ${ }^{28}$ Additionally, the complex structures fabricated by $3 \mathrm{D}$ printing can realize unique properties in piezoelectric sensors. Zhou et al. designed a unique kirigami pattern and 3D printed both electrodes and the piezoelectric BTO/P(VDF-TrFE) composite by DIW $^{29}$ (Figure $4 d$ ). The unique kirigami pattern realizes stretchability in the originally flexible PVDF-based material, and also eliminates the out-of-plane protrusion under stretching of the typical kirigami structure, enabling the pressing mode for kirigami-patterned piezoelectric gait sensor. Besides the new mechanical property, the piezoelectric property and sensing behavior can also be changed by complex 3D structures. Cui et al. have reported unique 3D structures by DLP printing of lead zirconate titanate nanoparticle (PZT)/poly(ethylene glycol) diacrylate (PEGDA) composite. ${ }^{30}$ With different 3D structures, the overall value and direction of the piezoelectric coefficient can be adjusted to realize a selfpowered tactile sensor with multidirectional sensing ability.

\section{Three-dimensional printed triboelectric sensors}

There are four functioning modes for triboelectric sensors: contact and separation, sliding, single electrode, and freestanding triboelectric layer modes, all of which require at least one layer of triboelectric material and at least one electrode. The choice of electrode material is the same as the previously discussed three types of sensors, and the triboelectric material includes a list of natural and synthesized materials, of which both the chemical structure and surface morphology can affect the surface charge distribution and therefore affect the triboelectricity. Triboelectric sensors are applied in the pneumatic soft robots and the soft robots that could detect the charge of the object they grasp. Recently, many 3D printable triboelectric materials were developed for tactile sensing applications. Parida et al. developed an extremely stretchable and self-healable PUA-based triboelectric nanogenerator that has a potential to be applied for robust and self-healing tactile sensor for soft robotics. ${ }^{31}$ In this work, both self-healing triboelectric material and self-healing electrodes are DIW printable. 3D printing also enables co-fabrication of triboelectric sensors with the soft robotics. Zhu et al. reported a commercial material MJ 3D printed pneumatic soft robotic finger, which acts as half of the triboelectric sensor for the contact and separation mode device ${ }^{6}$ (Figure 4e). Different patterns are printed on the finger, in which the cuboid pattern improves the bending curvature sensing behavior due to the large and uniform contact area. Similarly, Jin et al. reported an FDM-printed triboelectric sensors with TPU as conductive material that assembles on a 3D printed soft robotic gripper ${ }^{32}$ (Figure 4f). Two kinds of triboelectric sensors with different structures on the gripper work together to sense and combining with machine learning to recognize 16 kinds of common objects with recognition accuracy of 98.125 percent.

Combining with other techniques, 3D printing can create triboelectric tactile sensors with more interesting properties, hierarchical structures, and more functionalities. Qian et al. employed DIW printed cellulose on cold plate and freeze dried it after printing to form porous hierarchical structure for bifunctional sensing of motion and humidity. ${ }^{33}$ DIW with embedded copper wire is demonstrated by Tong et al. ${ }^{34}$ The co-axial filament-based triboelectric sensor can achieve realtime monitoring of organism swelling and speech recognition in the absence of sound.

\section{Conclusion}

In summary, we have discussed the 3D printing technique for four types of tactile sensors for soft robotics application, including piezoresistive, capacitive, piezoelectric, and triboelectric sensors. Three-dimensional printing can simplify the fabrication process, enable multimaterial co-deposition, and fabricate designed macrostructures that can affect the sensing performances. This makes it possible to develop multifunctional tactile sensors that can be integrated with different forms of soft robotics for complex tasks. In the future, the effect of $3 \mathrm{D}$ printing on the functionality of materials and the strain engineering of the device structures are suggested to be further studied, to make advantages of both structural and materials advances, in order to develop more adaptable, multifunctional tactile sensors for soft robotics. Printing tactile sensors together with readout circuits, actuators, transistors, energy harvesters and other devices and finally fabricating the whole soft robot in a single step without assembly is also desirable for the soft robotics community. The potential challenges for achieving this goal include the interface mechanical compatibility between different materials, and the noise or interference signal from neighboring or adjacent materials and structures, which can be solved by strain engineering and development of printable material systems.

\section{Acknowledgments}

This work was supported by the National Research Foundation Investigatorship (Award No. NRF-NRFI2016-05), and Campus for Research Excellence and Technological Enterprise (CREATE) that is supported by the National Research Foundation, Prime Minister's Office, Singapore.

\section{Data availability}

Data sharing not applicable to this article as no datasets were generated or analyzed during the current study.

\section{Conflict of interest}

On behalf of all authors, the corresponding author states that there is no conflict of interest.

\section{Open Access}

This article is licensed under a Creative Commons Attribution 4.0 International License, which permits use, sharing, adaptation, distribution and reproduction in any medium or format, as long as you give appropriate credit to the original author(s) and the source, provide a link to the Creative Commons 
licence, and indicate if changes were made. The images or other third party material in this article are included in the article's Creative Commons licence, unless indicated otherwise in a credit line to the material. If material is not included in the article's Creative Commons licence and your intended use is not permitted by statutory regulation or exceeds the permitted use, you will need to obtain permission directly from the copyright holder. To view a copy of this licence, visit http:// creativecommons.org/licenses/by/4.0/.

\section{References}

1. L. Zou, Novel tactile sensor technology and smart tactile sensing systems: A review. Sensors (Basel). 17(11), 2653 (2017)

2. M.I. Tiwana, S.J. Redmond, N.H. Lovell, A review of tactile sensing technologies with applications in biomedical engineering. Sensors Actuators A Phys. 179, 17 (2012)

3. Z.L. Wang, Triboelectric nanogenerators as new energy technology and selfpowered sensors - Principles, problems and perspectives. Faraday Discuss. 176, 447 (2014)

4. E. Davoodi, H. Fayazfar, F. Liravi, E. Jabari, E. Toyserkani, Drop-on-demand highspeed 3D printing of flexible milled carbon fiber/silicone composite sensors for wearable biomonitoring devices. Addit. Manuf. 32, 101016 (2020)

5. B. Shih, C. Christianson, K. Gillespie, S. Lee, J. Mayeda, Z. Huo, M.T. Tolley, Design considerations for 3D printed, soft, multimaterial resistive sensors for soft robotics. Front. Robot. A/6, 30 (2019)

6. M. Zhu, M. Xie, X. Lu, S. Okada, S. Kawamura, A soft robotic finger with selfpowered triboelectric curvature sensor based on multi-material 3D printing. Nano Energy 73, 104772 (2020)

7. A. Tuna, O.K. Erden, Y.D. Gokdel, B. Sarioglu, 3D printed capacitive pressure sensor with corrugated surface. Emerg. Non-CMOS Technol. II, 149 (2017)

8. S.S.K. Mallineni, Y. Dong, H. Behlow, A.M. Rao, R. Podila, A wireless triboelectric nanogenerator. Adv. Energy Mater. 8, 1 (2018)

9. H. Li, R. Li, X. Fang, H. Jiang, X. Ding, B. Tang, G. Zhou, R. Zhou, Y. Tang, 3D printed flexible triboelectric nanogenerator with viscoelastic inks for mechanical energy harvesting. Nano Energy 58, 447 (2019)

10. X. Zhou, K. Parida, O. Halevi, S. Magdassi, All 3D printed stretchable piezoelectric nanogenerator for self-powered sensor application. Sensors MDPI20, 1 (2020)

11. D.T. Pham, R.S. Gault, A comparison of rapid prototyping technologies. Int. J. Mach. Tools Manuf. 38, 1257 (1998)

12. S. Guo, K. Qiu, F. Meng, S.H. Park, M.C. McAlpine, 3D printed stretchable tactile sensors. Adv. Mater. 28, 1701218 (2017)

13. J.F. Christ, N. Aliheidari, P. Potschke, A. Ameli, Bidirectional and stretchable piezoresistive sensors enabled by multimaterial 3D printing of carbon nanotube/ thermoplastic polyurethane nanocomposites. Polymers (Basel) 11(1), 11 (2018)

14. X. Gan, J. Wang, Z. Wang, Z. Zheng, M. Lavorgna, A. Ronca, G. Fei, H. Xia, Simultaneous realization of conductive segregation network microstructure and minimal surface porous macrostructure by SLS 3D printing. Mater. Des. 178, 07874 (2019)

15. K. Huang, S. Dong, J. Yang, J. Yan, Three-dimensional printing of a tunable graphene-based elastomer for strain sensors with ultrahigh sensitivity. Carbon 143, 63 (2019)

16. Y. Jiang, Z. Liu, N. Matsuhisa, D. Qi, W.R. Leow, H. Yang, J. Yu, G. Chen, Y. Liu, C. Wan, Z. Liu, X. Chen, Auxetic mechanical metamaterials to enhance sensitivity of stretchable strain sensors. Adv. Mater. 30(12), 1706589 (2018)

17. P. Sensors, Z. Tang, S. Jia, X. Shi, B. Li, C. Zhou, Coaxial printing of silicone elastomer composite fibers for stretchable and wearable piezoresistive sensors. Polymers (Basel) 11(4), 666 (2019)

18. P. Wei, H. Leng, Q. Chen, R.C. Advincula, E.B. Pentzer, Reprocessable 3D-printed conductive elastomeric composite foams for strain and gas sensing. ACS Appl. Polym. Mater. 1, 885 (2019)

19. E. Davoodi, H. Montazerian, R. Haghniaz, A. Rashidi, S. Ahadian, A. Sheikhi, J. Chen, A. Khademhosseini, A.S. Milani, M. Hoorfar, E. Toyserkani, 3D-printed ultra-robust surface-doped porous silicone sensors for wearable biomonitoring. ACS Nano 14, 1520 (2020)

20. S. Peng, Y. Li, L. Wu, J. Zhong, Z. Weng, L. Zheng, Z. Yang, J. Miao, 3D printing mechanically robust and transparent polyurethane elastomers for stretchable electronic sensors. ACS Appl. Mater. Interfaces 12, 6479 (2020)

21. C.J. Hohimer, G. Petrossian, A. Ameli, C. Mo, P. Pötschke, 3D printed conductive thermoplastic polyurethane/carbon nanotube composites for capacitive and piezoresistive sensing in soft pneumatic actuators. Addit. Manuf. 34, 101281 (2020)
22. M. Ntagios, H. Nassar, A. Pullanchiyodan, W.T. Navaraj, R. Dahiya, Robotic hands with intrinsic tactile sensing via 3D printed soft pressure sensors. Adv. Intell. Syst. 2(6), 1900080 (2020)

23. Q. Zou, Z. Ma, S. Li, Z. Lei, Q. Su, Tunable ionic pressure sensor based on 3D printed ordered hierarchical mesh structure. Sensors Actuators A Phys. 308, $112012(2020)$

24. Z. Lei, Q. Wang, P. Wu, A multifunctional skin-like sensor based on a 3D printed thermo-responsive hydrogel. Mater. Horiz. 4, 694 (2017)

25. Y. Gao, G. Yu, T. Shu, Y. Chen, W. Yang, Y. Liu, J. Long, 3D-printed coaxial fibers for integrated wearable sensor skin. Adv. Mater. Technol. 4(10), 1900504 (2019)

26. M. Saari, B. Xia, B. Cox, P.S. Krueger, A.L. Cohen, E. Richer, Fabrication and analysis of a composite 3D printed capacitive force sensor. 3D Print. Addit. Manuf. 3, 137 (2016)

27. C. Lee, J.A. Tarbutton, Electric poling-assisted additive manufacturing process for PVDF polymer-based piezoelectric device applications. Smart Mater. Struct. 23, 095044 (2014)

28. H. Kim, F. Torres, D. Villagran, C. Stewart, Y. Lin, T.-L.B. Tseng, 3D Printing of $\mathrm{BaTiO}_{3} / \mathrm{PVDF}$ composites with electric in situ poling for pressure sensor applications. Macromol. Mater. Eng. 302(11), 1700229 (2017)

29. X. Zhou, K. Parida, O. Halevi, Y. Liu, J. Xiong, S. Magdassi, P.S. Lee, All 3D-printed stretchable piezoelectric nanogenerator with non-protruding kirigami structure. Nano Energy 72, 104676 (2020)

30. H. Cui, R. Hensleigh, D. Yao, D. Maurya, P. Kumar, M.G. Kang, S. Priya, X. Zheng, Three-dimensional printing of piezoelectric materials with designed anisotropy and directional response. Nat. Mater. 18, 234 (2019)

31. K. Parida, G. Thangavel, G. Cai, X. Zhou, S. Park, J. Xiong, P.S. Lee, Extremely stretchable and self-healing conductor based on thermoplastic elastomer for all-three-dimensional printed triboelectric nanogenerator. Nat. Commun. 10, 2158 (2019)

32. T. Jin, Z. Sun, L. Li, Q. Zhang, M. Zhu, Z. Zhang, G. Yuan, T. Chen, Y. Tian, X. Hou, C. Lee, Triboelectric nanogenerator sensors for soft robotics aiming at digital twin applications. Nat. Commun. 11, 5381 (2020)

33. C. Qian, L. Li, M. Gao, H. Yang, Z. Cai, B. Chen, Z. Xiang, Z. Zhang, Y. Song, All-printed 3D hierarchically structured cellulose aerogel based triboelectric nanogenerator for multi-functional sensors. Nano Energy 63, 103885 (2019)

34. Y. Tong, Z. Feng, J. Kim, J.L. Robertson, X. Jia, 3D printed stretchable triboelectric nanogenerator fibers and devices. Nano Energy 75, 104973 (2020)

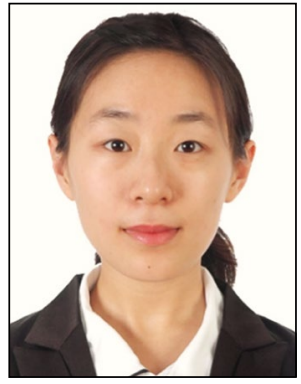

Xinran Zhou is a doctoral candidate in the School of Materials Science and Engineering at Nanyang Technological University, Singapore. She received her BS degree from the School of Materials Science and Engineering at Nanyang Technological University in 2017. Her research focuses on three-dimensional printing of piezoelectric materials for application in energy harvesting and soft electronics. Zhou can be reached by email at xrzhou@ntu.edu.sg.

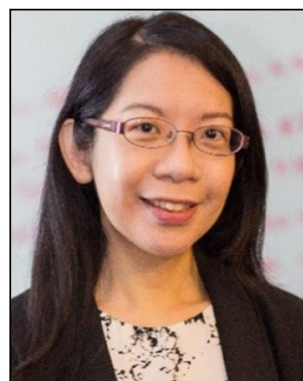

Pooi See Lee has been a full professor at the Nanyang Technological University, Singapore, since 2015. She joined the School of Materials Science and Engineering at Nanyang Technological University as an assistant professor in 2004 . She was promoted to tenured associate professor in 2009 Lee received her $\mathrm{PhD}$ degree from the National University of Singapore in 2002. Her awards include the Norman Hackerman Young Author from the Electrochemical Society in 2002, the National Research Foundation Investigatorship, and the Nanyang Research Excellence Award in 2016. Her research focuses on energy and electronics, flexible and stretchable devices, electrochemical devices, and human-machine interface. Lee can be reached by email at pslee@ntu.edu.sg. 